\title{
Innate Immunity Response to Tissue Engineering Templates: The Determinant
}

\author{
Marko Z. Radic ${ }^{1}$ and Gary L. Bowlin ${ }^{2}$ \\ ${ }^{1}$ Department of Microbiology, Immunology, and Biochemistry, The University of Tennessee Health Science Center, Memphis, USA
}

${ }^{2}$ Department of Biomedical Engineering, The University of Memphis and Joint University of Memphis-UTHSC-Memphis Biomedical Engineering Program, Memphis, USA

*Corresponding author: Gary L. Bowlin, Department of Biomedical Engineering, The University of Memphis Joint University of Memphis -UTHSC-Memphis Biomedical Engineering Program, Memphis, USA, Tel: 901-678-2670; Email: glbowlin@memphis.edu

Rec date: May 01, 2014, Acc date: May 2 2014, Pub date: May 3, 2014

Copyright: (C) 2014 Bowlin GL, et al. This is an open-access article distributed under the terms of the Creative Commons Attribution License, which permits unrestricted use, distribution, and reproduction in any medium, provided the original author and source are credited.

Keywords: Tissue engineering template; Innate immune system; Macrophage; Neutrophil; Platelet; Tissue regeneration

\section{Introduction}

An intense focus of the tissue engineering community is currently concentrated on the development of temporary, acellular tissue regeneration templates with an architecture and composition that will harness the body as a bioreactor to promote in situ formation of new tissues and organs. These templates are fabricated by numerous methods (e.g. electrospinning to decellularization) from a wide variety of synthetic and natural materials. Regardless, they all have one critical aspect in common: Immediately upon implantation, the innate immune system interacts with the template and initiates a sequence of multifaceted events that will be the ultimate determinant of template success (angiogenesis and regeneration) or failure (inflammation and fibrosis/scar tissue).

The innate immune system is the body's first line of defense to provide immediate, albeit fairly short-lived protection/defense from infections (i.e. bacteria), toxins, and other foreign substances (i.e. synthetic implanted biomaterial templates) in a non-specific manner against these agents that one would consider hazardous to tissues/ organs and homeostasis. In other words, the cellular components involved in the innate immune system recognize and respond to these entities in an immediate, generic fashion unlike the more complex and delayed acquired immune system. We know that biomaterial implants are immediately coated with blood plasma proteins followed by the adhesion of leukocytes (primarily neutrophils and monocytes from the blood stream). One looming and unanswered question currently remains $i$ What is the overall role of neutrophils in the preconditioning of the material/template and establishing the microenvironment to set the stage for the subsequent in tissue regeneration?

\section{Innate Immunity Cellular Components and Their Perceived Roles}

The primary cellular components of the innate immune system include mast cells, phagocytes (monocyte/macrophages, neutrophils and dendritic cells), basophils, eosinophils, Natural Killer cells (NK cells), and platelets. Neutrophils are highly mobile cells of the innate immune system which are recruited rapidly and in large quantities by chemotaxis to the site of inflammation/injury. Historically, these cells were thought of as the "suicidal killers" because they came in and their actions (release their granule contents with toxic substances against pathogens) were thought to be swift, followed by apoptosis and clearance by macrophages. Evidence now indicates this is inaccurate, and we really need to rethink and focus on the role of neutrophils in wound healing and the interaction with tissue engineering templates.
Particular attention should be directed at the temporal progression of events that neutrophils set into motion and that contribute to tissue healing/regeneration. The interaction between neutrophils and tissue regeneration templates must be regulated tightly because an inappropriate, prolonged neutrophil response can lead to extensive collateral tissue damage caused by the release of reactive oxygen species. Of particular note, the activated neutrophils provide the signals for the recruitment, activation, and differentiation of macrophages and dendritic cells [1]. Tissue macrophages and monocytes recruited from the blood stream (which differentiate into macrophages) are the large phagocytic cells involved in clearing large quantities of debris and bacteria. The macrophages also contribute to the subsequent tissue repair which is currently a major focus in the field of in situ, a cellular tissue engineering template regeneration. The dendritic cells present antigens to the T cells and thus act as the envoy between the innate and acquired immunes systems. The NK cells are important as part of the innate immune system due to their ability to kill virus infected and tumor cells. Finally, the eosinophils and basophils are similar to neutrophils in that they release compounds that aid in defense particularly against parasites but they may result in potential collateral tissue damage. As one of the cells that arrives instantly at the injury site, platelets appear to have three roles: 1) hemostasis (platelet plug), 2) direct binding via cell-surface receptors to various innate immune system cells and pathogens, and 3) the secretion of various cytokines and antimicrobial peptides that aid in modulating the innate response. It should be noted that we do not fully appreciate the degree to which cells of the innate immune system synergistically interact, nor do we know the precise nature of their multifaceted interactions. This knowledge is essential to understand the innate immune response to pathogens as well as tissue engineering templates. Most critically, platelets and neutrophils will be the first and most numerous cells of the innate immune system to interact with the template and set the stage for the subsequent events. Thus, these cells should be considered the true "maestros" for orchestrating the innate response to the implanted template. As such, they cannot continue to be ignored as the critical contributors to tissue engineering template success/failure.

\section{Innate Immunity as It Currently Relates to Tissue Engineering Template Regeneration}

There has been and continues to be a great deal of attention by researchers on macrophage, mast cells, and dendritic cells, with respect to their response due to the modification of biomaterial implants and their architectures. Historically, one thinks of implanted biomaterials/ templates and innate immunity interaction as inflammation leading to macrophages fusing to form multinucleated foreign-body giant cells and their contribution to fibrous encapsulation and failure [2]. From 
wound healing studies and other research, we know that this is not a necessary outcome as the macrophage phenotype exhibits plasticity that is regulated by the cellular microenvironment. The macrophage phenotype is now viewed as a continuum of functional states between two opposing endpoints. At one end is the M1 phenotype, embodied by classically activated macrophages, which are pro-inflammatory and microbicidal, while at the other end is the M2 phenotypes (multiple subsets) of alternatively activated macrophages that are immunomodulatory, angiogenic, regenerative, and poorly microbicidal. In normal injuries, the sequential appearance and relative balance of these two phenotypes plays a critical role in the phagocytosis of pathogens, the clearance of apoptotic cells, and the remodeling of injured tissues/implanted templates. In a seminal study of skin biopsies from human patients, gene expression was analyzed at early (day 1-2) and late (day 4-8) stages of wound healing. It was observed that the early stage included a mix of M1 and M2 markers, whereas the late stage displayed predominately M2 markers. This was viewed as a typical and necessary progression of debriding and healing macrophage phenotype distributions [3]. The recapitulation of this phenotype progression will be critical for tissue engineering templates because prolonged M1 activation will lead to tissue injury. Therefore, one must achieve the transition of M1s to M2s and maintain an appropriate relative M1: M2 ratio to facilitate proper tissue remodeling and angiogenesis after disinfecting and debriding a wound site. Unfortunately, this important insight into macrophage phenotype has haphazardly focused a great deal of the field on this single aspect of the innate immune response to implanted templates.

The classical view of the neutrophil functions during wound healing and/or template regeneration is now undergoing a reevaluation, in part due to an expansive knowledge of the role of neutrophils in cancer tumor biology. Only recently has the field of immunology began to appreciate the sophistication of neutrophils and the complexity of their function. This is primarily due to evidence that neutrophil granules release chemokines and cytokines that orchestrate the overall inflammation/immune response, including the priming of the macrophage response. Thus, it is high time to rethink the overall heterogeneity of the neutrophil populations in terms of phenotypic and functional profiles as well as their life-span and manner of cell death under pathological and physiological conditions. Extensive tumor biology studies teach us that Tumor Associated Neutrophils (TANs) constitute a large percentage of the immune cell infiltrate in solid tumors. These TANs have considerable plasticity which is regulated by the microenvironment, to date exhibiting two clear subsets (anti-tumor $\mathrm{N} 1$ and pro-tumor N2) that are analogues to the M1 and M2 macrophages phenotypes. This commonality should come as no surprise given the common origin/genesis of neutrophils and monocyte/macrophages [4]. The antitumor N1 population activates the immune system due to enhanced expression of cytokines and chemokines, lower levels of arginase, and a higher capacity to kill tumor cells [4]. The N2 population is characterized by the expression of higher levels of CXCR4, arginase, VEGF, and MMP-9 [4]. Thus, it is hypothesized that the appropriate design of a tissue engineering template architecture/composition will regulate the neutrophil response and tailor the desired response, leading to reproducible and successful template regeneration. An additional key contributing to potential success and a need for refocused attention is that the neutrophil's known robust angiogenic potential is expression of a large quantity of matrix metalloprotease 9 (MMP-9) which is uniquely released free of tissue inhibitor of metalloprotease 1 (TIMP-1). Neutrophils thus deliver TIMP-free MMP-9 as a potent, proangiogenic molecule [5]. An important recent discovery, the induction of neutrophil swarms or clusters by cell damage that is a consequence of injury, infection or trauma, will also demand attention with regard to its effects on neutrophil responses to tissue regeneration templates [6].

\section{Future Direction and Refocusing of the Field}

It is clear that there is still a great deal of research and understanding required about the innate immune response to implants. It is imperative that the research focus shift immediately to the initial phase and cellular components of the innate immune response to tissue engineering templates, i.e. the role and importance of neutrophils and platelets along with their synergistic interactions with engineered templates. More essentially, we must use all of this information to design the next generation of tissue regenerative templates that will promote the desired overall innate immune response. Currently, the field appears to be haphazardly working its way back from the final sequences of events to the initiation phase of the response. It should be clear now that one must orchestrate the entire innate immune response from the time of implantation, not just the later steps, to obtain a successful outcome (tissue regeneration) upon implantation of a tissue engineering template. This is especially true because the later stages, which are currently the focus of much research, are set in motion and dictated by the initial response of the platelets and neutrophils. Without this proposed shift in focus, we will continue to struggle to achieve a reproducible, successful outcome of tissue engineering templates. This is due to the basic fact that the entire, temporal innate immune response determines the success or failure of an implanted acellular template.

\section{References}

1. Kumar V, Sharma A (2010) Neutrophils: Cinderella of innate immune system. Int Immunopharmacol 10: 1325-1334.

2. McNally AK, Anderson JM (2011) Macrophage fusion and multinucleated giant cells of inflammation. Adv Exp Med Biol 713: 97-111.

3. Deonarine K, Panelli MC, Stashower ME, Jin P, Smith K, et al. (2007) Gene expression profiling of cutaneous wound healing. J Transl Med 5: 11.

4. Piccard H, Muschel RJ, Opdenakker G (2012) On the dual roles and polarized phenotypes of neutrophils in tumor development and progression. Crit Rev Oncol Hematol 82: 296-309.

5. Ardi VC, Kupriyanova TA, Deryugina EI, Quigley JP (2007) Human neutrophils uniquely release TIMP-free MMP-9 to provide a potent catalytic stimulator of angiogenesis. Proc Natl Acad Sci U S A 104: 20262-20267.

6. Lämmermann T, Afonso PV, Angermann BR, Wang JM, Kastenmüller W, et al. (2013) Neutrophil swarms require LTB4 and integrins at sites of cell death in vivo. Nature 498: 371-375. 